\title{
Article \\ Characterising the Mould Rectification Process for Designing Scoliosis Braces: Towards Automated Digital Design of 3D-Printed Braces
}

\author{
Inigo Sanz-Pena ${ }^{1}{ }^{1}$, Shanika Arachchi ${ }^{2}$, Dhammika Halwala-Vithanage ${ }^{3}$, Sanjaya Mallikarachchi ${ }^{4}$, \\ Jeewantha Kirumbara-Liyanage ${ }^{3}$, Alison McGregor ${ }^{5}$ (D) , Pujitha Silva ${ }^{4}$ and Nicolas Newell ${ }^{1, *(1)}$
}

1 Department of Mechanical Engineering, Imperial College London, London SW7 2AZ, UK; i.sanz-pena@imperial.ac.uk

2 Department of Applied Computing, University of Kelaniya, Kelaniya 11600, Sri Lanka; shanikami@kln.ac.lk

3 Sri Lanka School of Prosthetics and Orthotics, Rheumatology and Rehabilitation Hospital Ragama, Gampaha 11010, Sri Lanka; dhammika.halwala-vithanage.2020@uni.strath.ac.uk (D.H.-V.); schslspo@health.gov.lk (J.K.-L.)

4 Center for Biomedical Innovation, University of Moratuwa, Moratuwa 10400, Sri Lanka; 140385u@uom.lk (S.M.); psilva@uom.lk (P.S.)

5 Department of Surgery and Cancer, Imperial College London, London W12 0BX, UK; a.mcgregor@imperial.ac.uk

* Correspondence: n.newell09@imperial.ac.uk

Citation: Sanz-Pena, I.; Arachchi, S.; Halwala-Vithanage, D.; Mallikarachchi, S.; KirumbaraLiyanage, J.; McGregor, A.; Silva, P.; Newell, N. Characterising the Mould Rectification Process for Designing Scoliosis Braces: Towards Automated Digital Design of 3D-Printed Braces. Appl. Sci. 2021, 11, 4665. https:// doi.org/10.3390/app11104665

Academic Editor: Francisco Cavas Martínez

Received: 12 April 2021

Accepted: 18 May 2021

Published: 19 May 2021

Publisher's Note: MDPI stays neutral with regard to jurisdictional claims in published maps and institutional affiliations.

Copyright: (c) 2021 by the authors. Licensee MDPI, Basel, Switzerland. This article is an open access article distributed under the terms and conditions of the Creative Commons Attribution (CC BY) license (https:/ / creativecommons.org/licenses/by/ $4.0 /)$.

\begin{abstract}
The plaster-casting method to create a scoliosis brace consists of mould generation and rectification to obtain the desired orthosis geometry. Alternative methods entail the use of $3 \mathrm{D}$ scanning and CAD/CAM. However, both manual and digital design entirely rely on the orthotist expertise. Characterisation of the rectification process is needed to ensure that digital designs are as efficient as plaster-cast designs. Three-dimensional scans of five patients, pre-, and post-rectification plaster moulds were obtained using a Structure Mark II scanner. Anatomical landmark positions, transverse section centroids, and 3D surface deviation analyses were performed to characterise the rectification process. The rectification process was characterised using two parameters. First, trends in the external contours of the rectified moulds were found, resulting in lateral tilt angles of $81 \pm 3.8^{\circ}$ and $83.3 \pm 2.6^{\circ}$ on the convex and concave side, respectively. Second, a rectification ratio at the iliac crest $(0.23 \pm 0.04$ and $0.11 \pm 0.02$ on the convex and concave side, respectively) was devised, based on the pelvis width to estimate the volume to be removed. This study demonstrates that steps of the manual rectification process can be characterised. Results from this study can be fed into software to perform automatic digital rectification.
\end{abstract}

Keywords: 3D scanning; adolescent idiopathic scoliosis; digital design; orthosis design; plastercasting; scoliosis brace; sculpting software

\section{Introduction}

Adolescent idiopathic scoliosis (AIS) is an abnormal curvature of the spine that occurs in 1.7 to $2.9 \%$ of the general population (for AIS with a Cobb angle over $10^{\circ}$ ) [1-5]. Brace treatment is the most common non-surgical intervention for the management of AIS. It involves fitting a low-profile spinal orthosis around the patient's torso and pelvis with the aim of controlling curve progression. Traditionally, scoliosis braces are either created using a patient-specific plaster-cast or by using prefabricated modules that fit the patient geometry [6]. Currently, the most widely used braces for the treatment of AIS are thoracic lumbar sacral orthosis (TLSO) that have high patient acceptance and tolerance [7]. Although there is controversy surrounding the efficacy of bracing AIS patients, TLSOs have been found to decrease the risk of curve progression and can protect against the need for surgery in skeletally immature AIS patients wearing a brace with high compliance [8-14]. 
The design of a plaster mould TLSO is divided in two steps. First, the creation of a positive mould from the cast of the patient's torso and, second, the rectification of the positive mould [15]. The rectification of the positive mould involves both removing and adding plaster in areas of the torso and pelvis to generate the desired mould geometry from which the brace shape is thermoformed. The rectification process is based on the orthotist's experience to achieve optimal in-brace spinal correction. Despite the wide use of this technique, there are downsides of manual mould rectification in that casting requires a significant amount of materials, tools, and time [16]. On average, it takes $60 \mathrm{~min}$ to cast a patient [17]. Furthermore, the geometry of the rectified mould is highly dependent on the orthotist expertise, and the manufacturing process involves the use of large equipment [18].

Recent technological advances have given the opportunity for alternative approaches to manual casting and rectification to be developed. These include employing computeraided design/computer-aided manufacturing (CAD/CAM) and 3D imaging to generate a virtual geometry of the patient's torso $[19,20]$. Three-dimensional imaging approaches, such as 3D scanning, present a promising technology for digital design and biomedical modelling applications using reverse engineering methods [21]. These methods can be used for digital rectification [22], positive mould manufacturing [15], and brace design [19,23,24], resulting in a reduction in the time required to generate a brace by $50 \%$ [18]. Several studies have compared braces developed using the plaster-casting method against CAD/CAM braces, finding either no significant difference $[15,22,25,26]$ or even better in-brace Cobb angle correction when using CAD/CAM methods [24].

The prospects of implementing a digital design process can go beyond the improvement of the geometry acquisition phase eliminating the need for casting. Manufacturing processes using additive manufacturing techniques present a promising solution to study the use of alternative materials to enhance the functionality, mechanical properties of the design [27], and use recycled materials [28]. Digital design of TLSOs allows for adaption of the design to create a new brace for the same patient when required by skeletal growth, compared to the plaster-casting method, for which a new cast must be completed to obtain the patient's geometry.

Despite the implementation of CAD/CAM methods in the design process, the rectification process still requires input from an orthotist to ensure an effective brace design. This involves the orthotist using rectification software, adding, or removing material digitally. Although the input of the orthotist is likely to always be required, semi-automation of this process may lead to a more efficient and repeatable design processes. Before this is possible, a detailed analysis of the manual rectification process is required. Therefore, the aim of this study is to determine whether it is possible to characterise the manual rectification process for the plaster-casting method. This would represent a step towards a fully automated CAD rectification process to generate more effective biomechanical brace designs and improve efficiency of the design process towards additive manufacturing of TLSOs.

\section{Materials and Methods}

\subsection{Patient Population, Scanner, and Mesh Generation Software}

A total of 5 AIS patients (2 left thoracolumbar and 3 right thoracolumbar curves), who had been prescribed TLSO Boston brace treatment at the Sri Lanka School of Prosthetics and Orthotics, Rheumatology and Rehabilitation Hospital Ragama, Gampaha, Sri Lanka, were recruited for this study. Posteroanterior (PA) X-rays of the patients were taken as part as the normal clinical process and not for the specific purpose of this study. The mean age of the patients was $13.7 \pm 1.8$ years (mean \pm standard deviation), ranging from 11-16 years old. The mean Risser sign was $1.8 \pm 1.6$, with values from $0-4$ and an average Cobb angle of $31.2^{\circ} \pm 11.2^{\circ}$, varying from $19^{\circ}-43^{\circ}$. The apical vertebrae were located from T12-L2. Approval from the SIDCER ethics review committee from the Faculty of Medicine, University of Kelaniya, Ragama, Sri Lanka, FWA00013225 (REF. P/08/02/2020) was obtained. The study was initiated after obtaining written consent from the patients' parents/guardians. 
A low-cost three-dimensional scanner Mark II (Occipital Inc., Boulder, CO, USA) was used in combination with an iPad Mini $5^{\text {th }}$ Gen (Apple Inc., Cupertino, CA, USA). The mesh generation software used was the Scanner application (Occipital Inc. Boulder, CO, USA). The selected scanner and mesh generation software demonstrated suitable accuracy and repeatability with maximum mean deviations within $1.7 \mathrm{~mm} \pm 3.6 \mathrm{~mm}$ on the torso of a standing subject. The maximum mean deviations were determined in a preliminary study testing different mesh generation software from a set of three torso scans.

\subsection{Scanning of Patients and Moulds}

Sets of 3D scans were obtained for each patient's body and plaster-cast moulds following their appointment at the clinic for a TLSO. Two different investigators performed the scans in a randomised order on the same day, walking around the patient maintaining a distance between 30-60 cm using floor markers as a reference, and ensuring the scanner was perpendicular to the patient's surface. The scans started all at the same point, in front of the patient and moving in the clockwise direction from the patient's point of view. The lighting of the environment was kept uniform to avoid any inaccuracies caused by changes in brightness. The patients were asked to stand with their arms raised and flexed anteriorly away from the body during the scanning process.

\section{Patient Geometry, Pre-Rectification, and Post-Rectification Mould Scans}

White spherical markers were placed over anatomical landmarks deemed important by the orthotists involved in the study for the design of a scoliosis brace and based on previous surface topography studies that have used markers to evaluate AIS [29-32]. The location of the markers used in this study are shown in Figure 1. The markers placed at the left and right angulus inferior scapulae are L-AI and R-AI, respectively. The CLAV marker is located at the manubrium, and the STRN marker is placed at the xiphisternal joint. On the anterior side of the pelvis markers, R-ASIS and L-ASIS correspond to the right and left anterior superior iliac spine, respectively, while R-IC1 and L-IC1 are located at the tubercle of the iliac crest on the right and left side. On the posterior side of the pelvis, R-PSIS and L-PSIS are located at the right and left posterior superior iliac spine. Lastly, R-TRO and L-TRO mark the right and left greater trochanter. Placement of the markers was performed by an experienced orthotist. Manual measurements of anatomical distances were obtained using a vernier calliper with a resolution of $0.02 \mathrm{~mm}$.

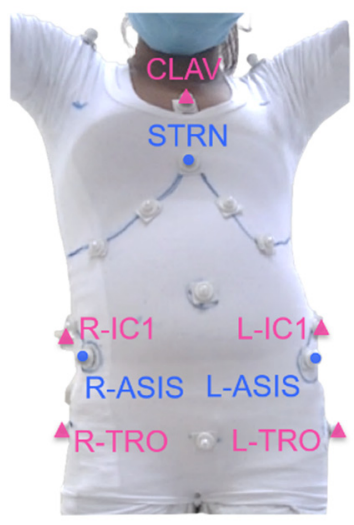

(a)

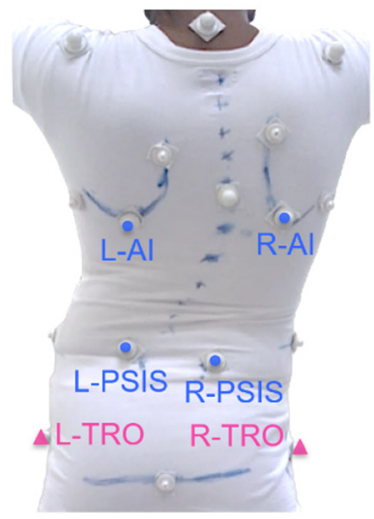

(b)

Figure 1. (a) Anterior and (b) posterior view of an AIS patient with spherical markers placed at anatomical landmarks. Magenta markers (triangle) are only placed on the patient's body, and blue markers (circle) represent markers placed on the patient's body, as well as the pre- and postrectification moulds.

The patients' moulds created using the plaster-casting method were scanned before and after the rectification process. Following the markers placed on the patient's torso, 
seven spherical markers (blue markers (circle) in Figure 1) were placed at the main anatomical landmarks on the pelvis and sternum on the anterior side (R-ASIS, L-ASIS, and STRN) (Figure 1a) and at the scapulae and pelvis on the posterior side (L-AI, R-AI, L-PSIS, and R-PSIS) (Figure 1b). These seven anatomical landmarks are used as references by the orthotist during the plaster rectification process and have also been found in a previous study to be the most common locations for evaluating AIS in surface topography studies [33].

\subsection{Characterisation of the Casting and Rectification Process}

The characterisation of the rectification strategies followed by the orthotist during the plaster-casting method was divided into three analyses. The first two focused on strategies that correct postural changes in the torso during the mould design phases. The third analysis focused on volume rectifications of the plaster mould (addition and removal of material), made to accommodate the pressure pads and define the brace shape. Each of these are discussed in more detail in the following sections.

\subsubsection{Alignment of the Scans}

To allow for comparison, scans were aligned using the pelvis markers of the postrectification mould as reference points. Pelvis markers (R-ASIS and L-ASIS) were chosen since the distance between them is maintained by the orthotist during the rectification process using a manual measurement taken from the patient's pelvis before the casting process as a reference. A preliminary investigation measured the distances digitally and compared them to the manual values. First, the post-rectification mould was oriented with the pelvis in a neutral position using the PA X-ray of the patient (Figure 2a). Second, depending on the type of curve of the patient (left or right), the marker on the concave side was chosen as the alignment reference (Figure $2 b$ ). Third, the patient's body and pre-rectification mould scans were aligned in the sagittal, coronal, and transverse plane to the reference post-rectification mould scan. Furthermore, the anatomical landmark distances measured from the patient's body and mould scans were compared to the manual measurements to verify the distance between markers in the scans during the different brace design phases for the casting method.

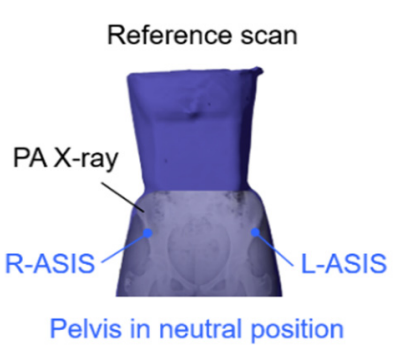

(a)

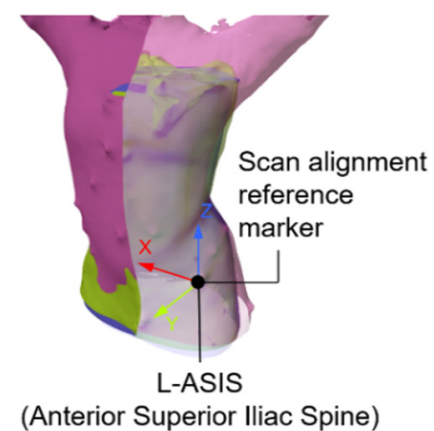

(b)

Figure 2. Alignment of the different scans obtained during the patient assessment and rectification process. (a) The post-rectification mould is used as the reference scan for alignment, oriented in a neutral pelvis position using the PA X-ray from the patient and the pelvis markers R-ASIS and L-ASIS located at the anterior superior iliac spine. (b) Isometric view of the three scans (patient's body, pre-, and post-rectification mould) aligned using the L-ASIS marker as the orientation reference for a right curve patient.

\subsubsection{Anatomical Landmark Positions}

Changes in anatomical landmark positions (ALPs) between the patient and postrectification mould scans were analysed using Blender software (Figure 3). The ALPs were obtained in 3D space using the patient's body scan centre of mass as the reference coordinate system for the marker positions, to determine the postural changes on the post- 
rectification mould. The ALPs were analysed in the coronal plane in both the medial/lateral $(\Delta \mathrm{X})$ and cranial/caudal direction $(\Delta \mathrm{Z})$ and in the sagittal plane in the anterior/posterior direction $(\Delta \mathrm{Y})$ (Figure $3 b)$. Changes in ALPs were analysed in combination with the patient's Cobb angle to investigate whether there was any correlation between the degree of the curvature and the position variations between the post-rectification mould and the patient's body scan.

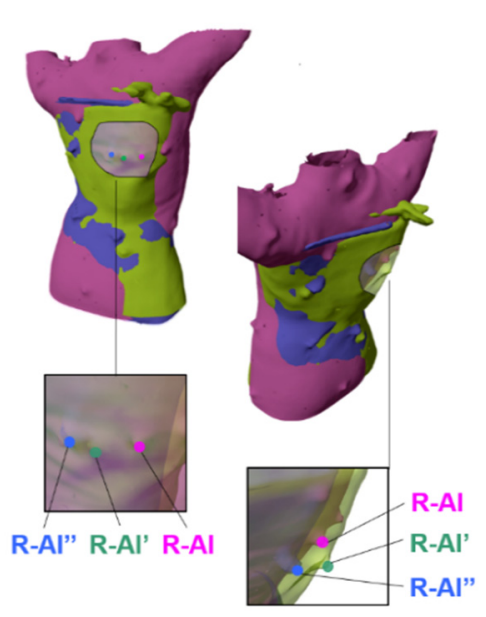

(a)

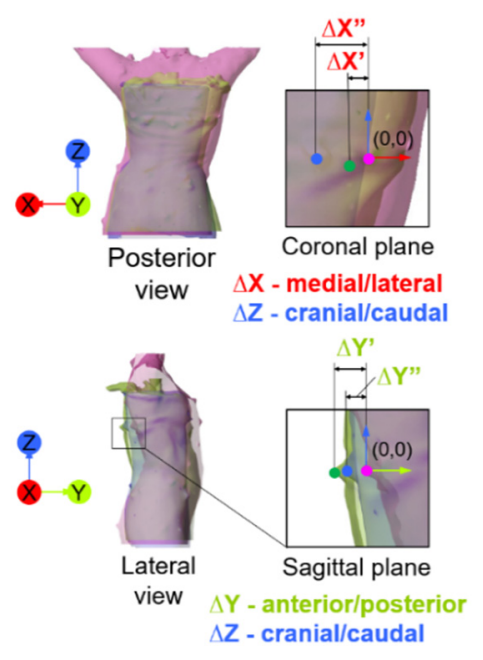

(b)

Figure 3. Anatomical landmark position (ALP) analysis. (a) The isometric views of the three scans aligned showing the location of marker right angulus inferior scapulae (R-AI) for the patient's scan (R$\mathrm{AI})$, pre-rectification mould scan (R-AI'), and post-rectification mould scan (R-AI"). (b) Posterior (top) and lateral (bottom) view of the three scans aligned and measure of ALP differences in the coronal and sagittal plane. The $X$-axis represents the medial/lateral direction; $Y$-axis is the anterior/posterior direction and Z-axis the cranial/caudal direction.

\subsubsection{Surface Centroids}

Transverse sections were made through the torso and pelvis on the patient's body, pre-, and post-rectification mould scans in the cranial/caudal direction. Sections for each patient were obtained at the level of L-ASIS, STRN, and CLAV marker positions corresponding to anatomical landmarks of the patient and a section at the middle section between STRN and L-ASIS (MID) (Figure 4a). For each transverse section, the centroid position of the external contour was obtained using Blender software. The centroid at the pelvis represented the reference coordinate system for the analysis. The origin was located equidistant between the pelvis marker locations R-ASIS and L-ASIS in the coronal plane, and between L-ASIS and L-PSIS in the sagittal plane (Figure 4a). The centroid location of transverse sections of the patient and moulds scans were compared to the centroid generated at the pelvis section. Deviations of the centroid location from the reference system were analysed between the patient's body, pre-, and post-rectification scans (Figure $4 \mathrm{~b}$ ). Deviations in the anterior/posterior direction are represented in the $Y$-axis and deviations in the medial/lateral direction in the $X$-axis. 
(a)

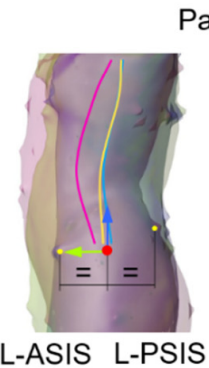

Patient 5

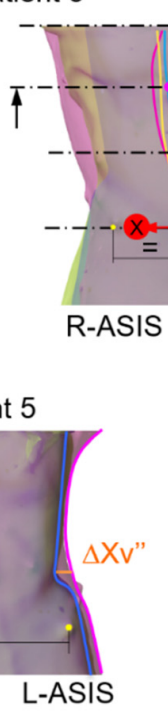

(c)

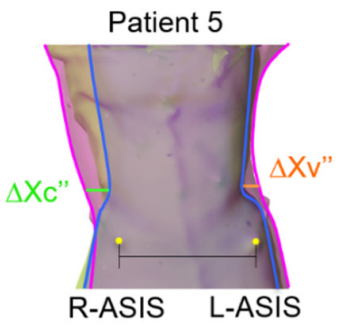

CLAV

STRN (b)

$$
\text { Transverse plane }
$$
section at STRN

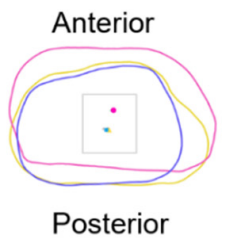

Concave side tilt

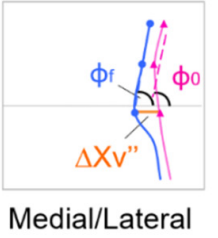

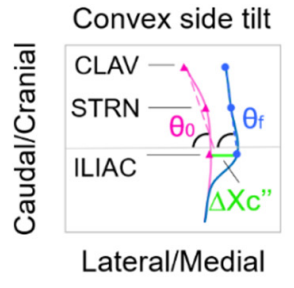

Rectification distance $\mid \triangle X_{C} "$ - convex side at the iliac crest transverse plane $\Delta X v^{\prime \prime}$ - concave side

Ratio $\mid \begin{aligned} & r X c=\Delta X c " / \text { (R-ASIS to L-ASIS) } \\ & r X v=\Delta X v^{\prime \prime} / \text { (R-ASIS to L-ASIS) }\end{aligned}$ $r X v=\Delta X v " /(R-A S I S$ to L-ASIS)

(d)

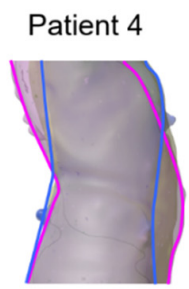

Posterior side sagittal tilt

Anterior side sagittal tilt
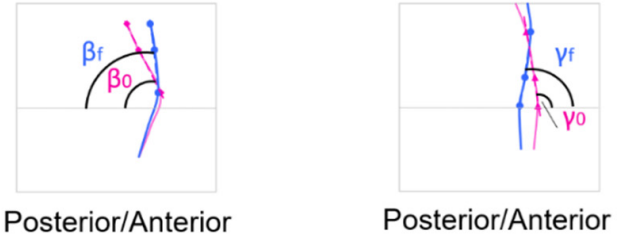

-Patient's body

- Pre-rectification mold —Post-rectification mold

Figure 4. Surface centroids and 3D surface deviation analyses. (a) The centroid trajectories of the body, pre-, and post-rectification mould scans from patient five. Dashed lines represent the transverse sections at marker level (CLAV, STRN, and L-ASIS). MID is a section at equal distance between STRN and L-ASIS. (b) External contours and centroids for the STRN section of patient five. (c) External lateral contours of patient's five body and post-rectification mould scan on the convex and concave side with their corresponding tilt angles and rectification distances at the iliac crest transverse plane ( $\Delta \mathrm{Xc}^{\prime \prime}$ and $\left.\Delta \mathrm{Xv}^{\prime \prime}\right)$. (d) External anterior and posterior contours of patient's four body and post-rectification mould scan with their corresponding tilt angles.

\subsubsection{Three-Dimensional Surface Deviations}

Volumetric differences were evaluated using 3D surface deviation maps obtained between the patient's body and post-rectification scans for each patient using CloudCompare. The 3D surface deviation maps were used to locate the largest rectification areas on the mould. Maximum deviations from the largest rectification areas were obtained in the anterior/posterior direction and medial/lateral direction, corresponding to the transverse planes located at the level of anatomical landmarks at the torso (CLAV, STRN, and superiorly of the iliac crest (ILIAC)) and pelvis (R-ASIS and R-TRO).

External contours of the patient's body and post-rectification scans were generated for the lateral sides on the coronal plane and the anterior/posterior sides for the sagittal plane (Figure 4c,d). The contours were used to analyse the rectification changes based on the contour deviations in the medial/lateral and anterior/posterior direction and the torso contour tilt in the coronal and sagittal plane. Tilt angles of the post-rectification mould and the patient's body scans were obtained from linear interpolation of the contour curves, using three points intersecting transverse sections determined by anatomical landmarks (CLAV, STRN, and ILIAC). A ratio was defined between the rectification distance at the 
ILIAC transverse section (waist level) and the pelvis width (R-ASIS to L-ASIS distance). The ratios on the convex $(\mathrm{rX})$ and concave side $(\mathrm{rXV})$ indicate the waist reduction on each side divided by the pelvis width (Figure 4c).

\section{Results}

\subsection{Anatomical Landmark Positions}

Deviations of each marker were represented using box plots in the medial/lateral $\left(\Delta X^{\prime \prime}\right)$ (Figure $\left.5 a\right)$, anterior/posterior $\left(\Delta Y^{\prime \prime}\right)$ (Figure $\left.5 b\right)$, and cranial/caudal direction $\left(\Delta Z^{\prime \prime}\right)$ (Figure 5c). The results showed no direct relationship between the Cobb angle and the ALP displacement in the medial/lateral direction.

$$
\text { (a) }
$$
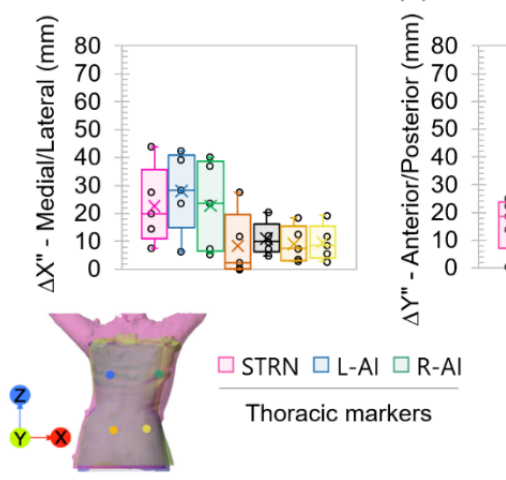

(b)

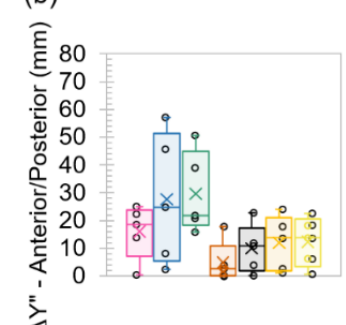

(c)

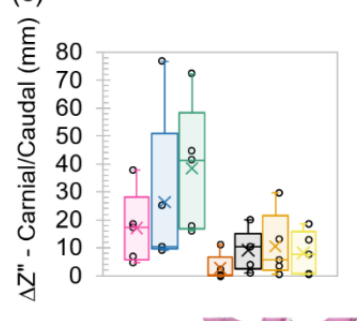

Figure 5. ALP deviations between the patient's body and the post-rectification mould. (a) represents the ALP deviations in the medial/lateral direction $\left(\Delta X^{\prime \prime}\right),(\mathbf{b})$ represents the ALP deviations in the anterior/posterior direction $\left(\Delta Y^{\prime \prime}\right)$, and (c) represents the ALP deviations in the cranial/caudal direction $\left(\Delta \mathrm{Z}^{\prime \prime}\right)$. STRN, L-AI, and R-AI are at the thoracic level on the anterior (STRN) and posterior (L-AI and R-AI) sides, while L-ASIS, R-ASIS, L-PSIS, and R-PSIS are the anterior (L-ASIS and R-ASIS) and posterior (L-PSIS and R-PSIS) sides of the pelvis. Mean deviations are represented by a cross, while median deviations are represented by a horizontal line. Top and bottom whiskers represent the maximum and minimum deviations.

\subsection{Surface Centroids}

Figure 6 represents the centroid coordinate results at four transverse sections of the scans, as well as the centroid trajectories in the coronal (Figure 6e) and sagittal (Figure 6f) planes.
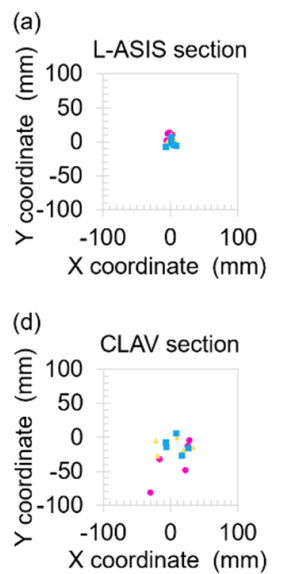
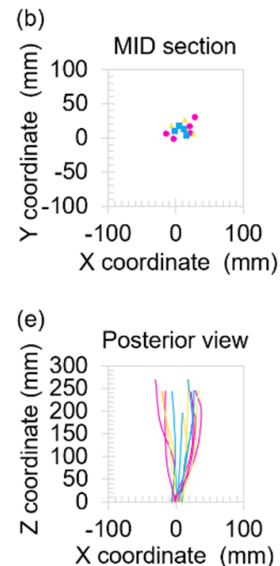
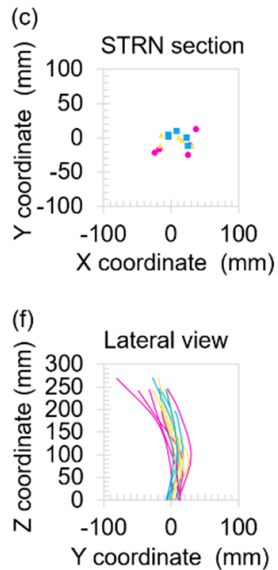

—Patient's body —Pre-rectification mold —Post-rectification mold

Figure 6. Centroid locations of the body scan, pre-, and post-rectification mould scans for the different patients. (a-d) are the coordinates of the centroids at each transverse section for all the patients. $(\mathbf{e}, \mathbf{f})$ are the centroid trajectories from the posterior and lateral view, respectively. 


\subsection{Three-Dimensional Surface Deviations}

Volumetric differences between the post-rectification mould and patient's body scans are represented using anterior, posterior, and lateral views of the 3D surface deviation plots (Figure 7). Box plots from the maximum deviation areas along transverse sections are represented in the medial/lateral $(\Delta \mathrm{X})$ and anterior/posterior $(\Delta \mathrm{Y})$ direction. Lateral deviations are compared on the convex and concave sides of the curve (Figure 7f,g, respectively).

(a)

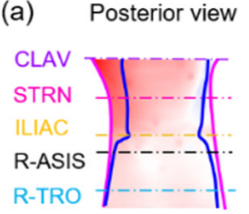

(b)<smiles>C=CC(C)C</smiles>

(c)<smiles>C=CCCCCCC</smiles>

(d)
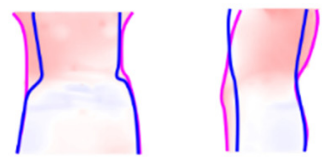

(e)
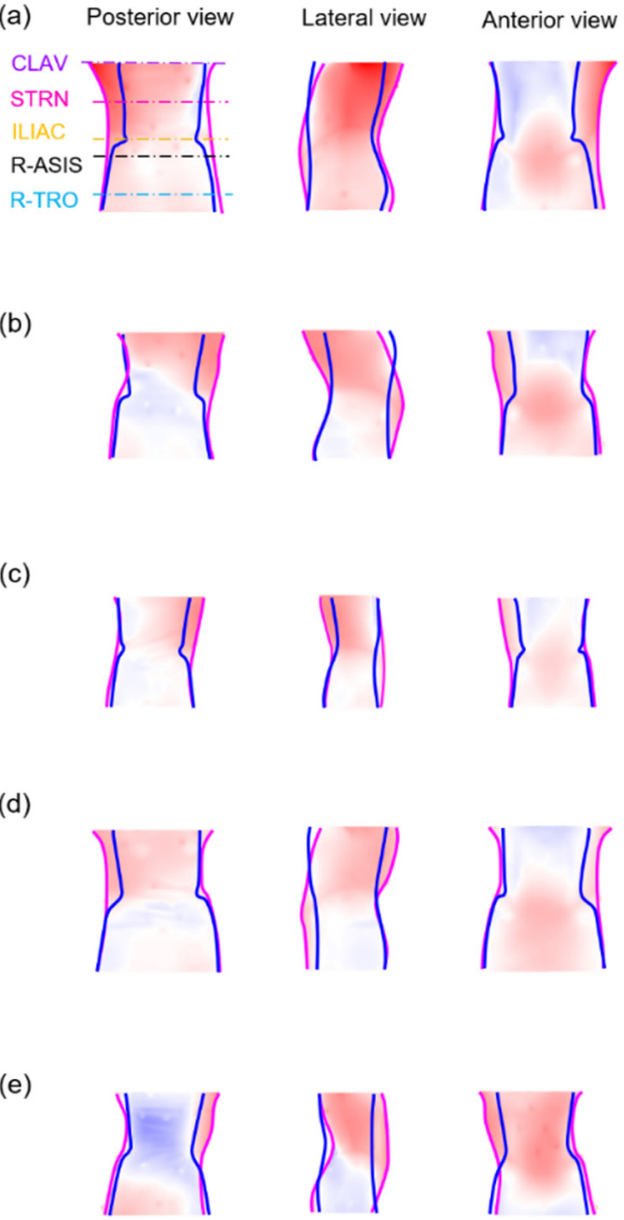

$\mathrm{mm}$
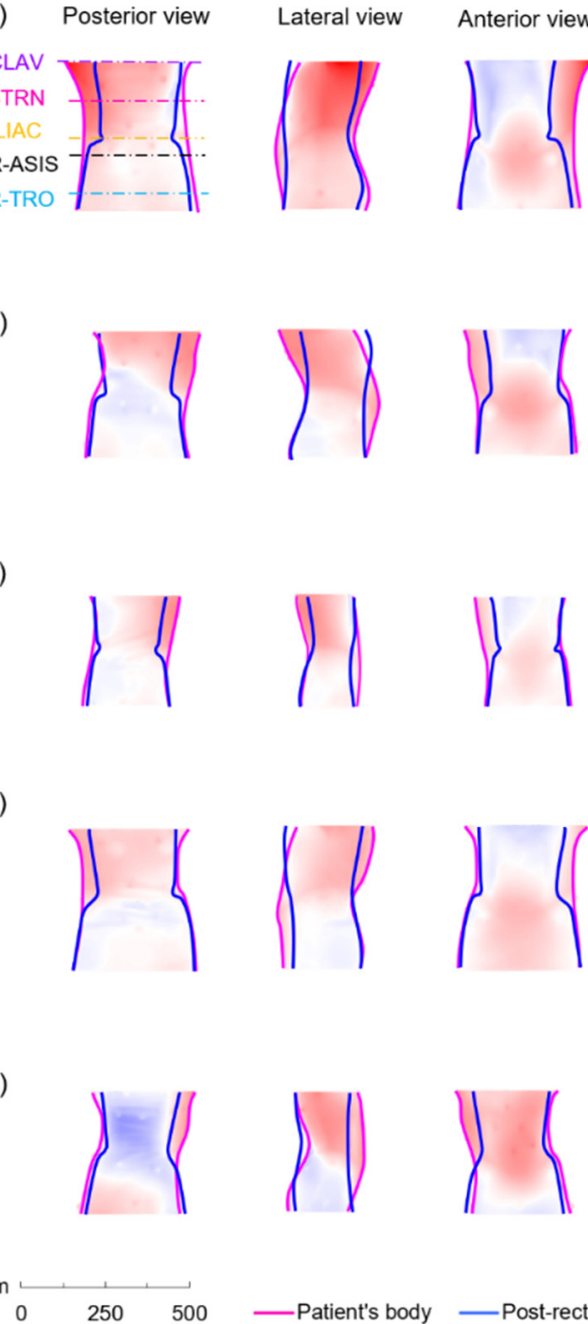
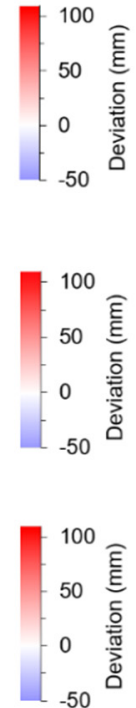

(f)

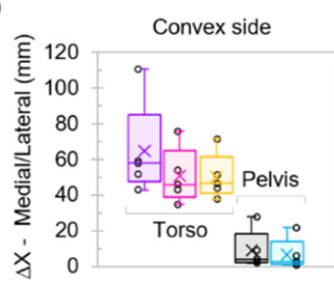

(g)

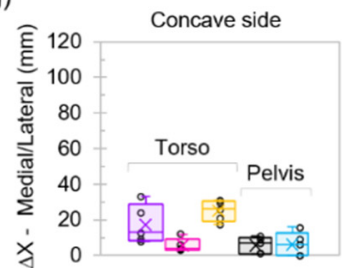

(h)

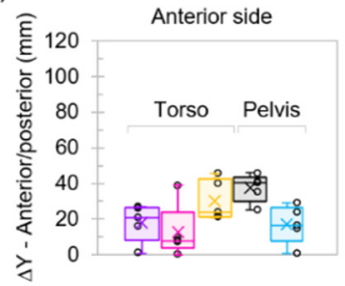

(i)

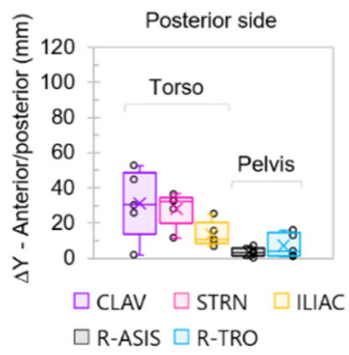

Figure 7. Three-dimensional surface deviation maps between the post-rectification mould and the patient's body for each patient (a-e) and box plots of maximum positive deviations at transverse sections (CLAV, STRN, ILIAC, R-ASIS, and R-TRO) (f-i). The deviation scales from the 3D surface maps represent the addition of material to the mould with respect to the patient's body and removal of material. Positive deviations represent removal of material from the plaster mould, while negative deviations represent adding material. (f) The maximum deviations in the medial/lateral direction ( $X$-axis) for all the patient's in the convex side and (g) concave side. (h) are the maximum deviations in the anterior/posterior direction ( $Y$-axis) for all the patient's in the anterior side and (i) posterior side. The maximum mean deviations are represented by a cross within the box plots, while maximum median deviations are represented by a horizontal line. Top and bottom whiskers represent the maximum and minimum deviations. Transverse sections CLAV, STRN, and ILIAC are located at the torso level and R-ASIS and R-TRO at the pelvis level.

External contours obtained from both the patient and post-rectification scans in the coronal plane are shown in Figure 8a,b, and in the sagittal plane in Figure 8c,d. Rectification ratios obtained in the medial/lateral direction at the iliac crest level (ILIAC) are shown in 
Figure 8i, found from the convex and concave side contours (Figure 8e,f). Furthermore, lateral and sagittal tilt angles on the convex and concave side ( $\theta$ and $\phi$, respectively) and posterior and anterior contours ( $\beta$ and $\gamma$, respectively) are shown in Figure $8 \mathrm{~g}, \mathrm{~h}$. Changes in tilt angles between the patient's body and post-rectification scans are shown in Figure 8j,k.

(a)

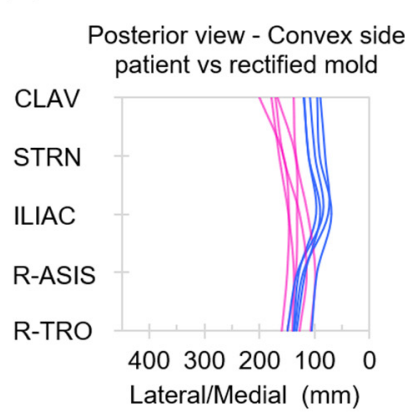

(e)

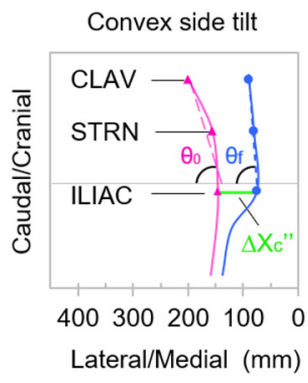

(b)

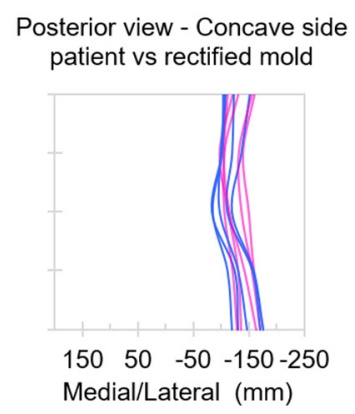

(f)

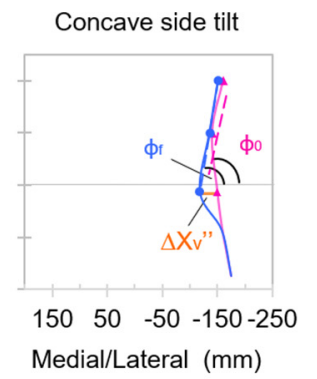

(c)

Lateral view - posterior side patient vs rectified mold

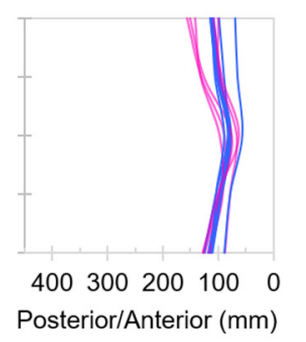

(g)

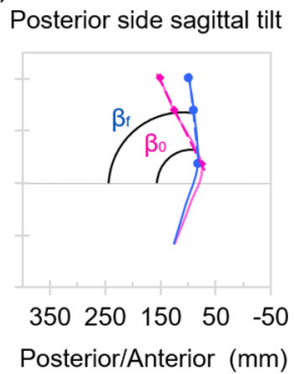

(d)

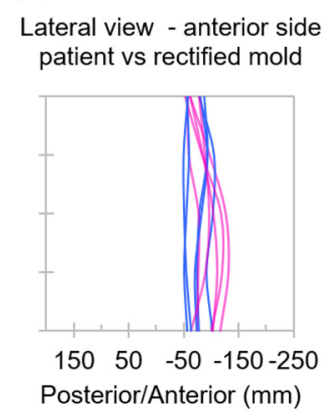

(h)

Anterior side sagittal tilt

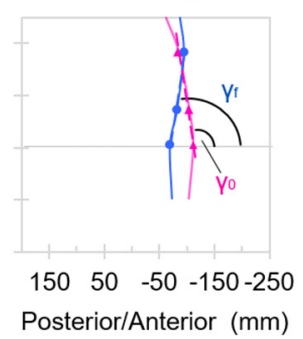

—Patient's body —Post-rectification mold

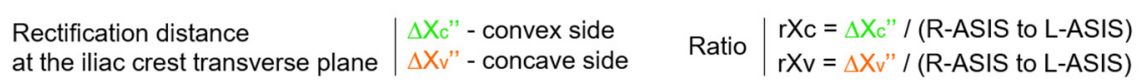

(i)

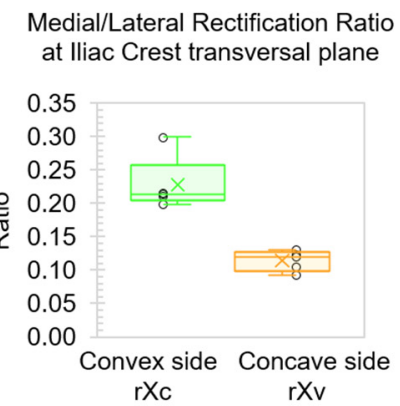

(j)

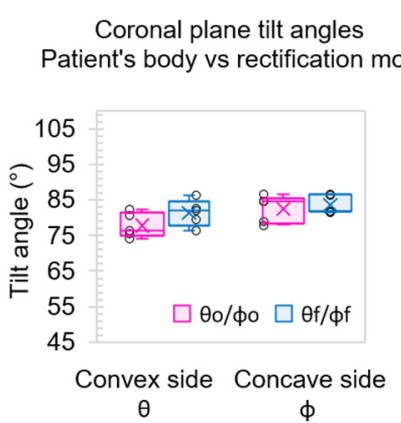

(k)

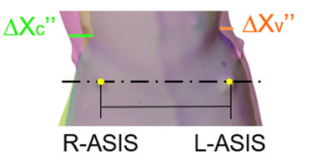

Sagittal plane tilt angles Patient's body vs rectification mold

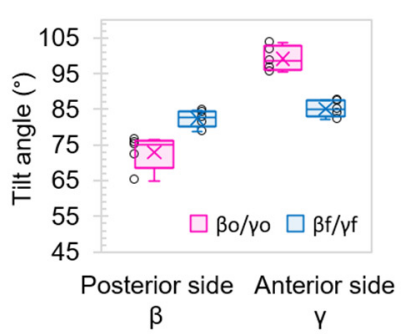

Figure 8. External contours of each patient's body and post-rectification scans generated for the lateral sides on the coronal plane and the anterior/posterior sides on the sagittal plane. (a) are the lateral contours on the convex side for the different patients and $(\mathbf{b})$ on the concave side. $(\mathbf{c}, \mathbf{d})$ are the posterior and anterior contours, respectively. $(\mathbf{e}, \mathbf{f})$ show the method to obtain the rectification distances at the ILIAC level $\left(\Delta \mathrm{Xc}_{\mathrm{c}^{\prime \prime}}, \Delta \mathrm{Xv}_{\mathrm{v}}{ }^{\prime \prime}\right)$ and tilt angles from the lateral contours of the patient's body and post-rectification mould on the convex $(\theta \mathrm{o} / \theta \mathrm{f})$ and concave sides $(\phi \mathrm{o} / \phi \mathrm{f})$, respectively. $(\mathrm{g}, \mathrm{h})$ show the posterior $(\beta \mathrm{o} / \beta \mathrm{f})$ and anterior tilt angles $\left(\gamma_{\mathrm{o}} / \gamma \mathrm{f}\right)$. (i) Box plot of the rectification ratios found between the rectification distance at the ILIAC level and the patient's width (R-ASIS to L-ASIS distance) for the convex $\left(r X_{c}\right)$ and concave $\left(r X_{v}\right)$ sides. (j,k) Box plots showing tilt angles on the coronal and sagittal plane for all the patients, respectively.

\section{Discussion}

This is the first study to attempt the characterisation of the plaster-casting process for the design of TLSO Boston braces. We analysed the differences between patient body scans 
and pre- and post-rectification moulds by investigating changes in anatomical landmark positions, centroid locations, and 3D surface deviation maps.

\subsection{Anatomical Landmark Position Analysis}

Deviations of ALP from the post-rectification mould with respect to the patient's body showed the lowest mean deviations at the anterior pelvis markers, with mean deviations from $2.9 \pm 4.8 \mathrm{~mm}$ (Figure $5 \mathrm{c}$ ) to $8.5 \pm 11.7 \mathrm{~mm}$ (Figure 5a) at L-ASIS and from $9.3 \pm 7.3 \mathrm{~mm}$ (Figure 5c) to $11 \pm 10 \mathrm{~mm}$ at R-ASIS (Figure 5a). This could be influenced by the use of the anterior pelvis markers as the alignment reference for the scans. However, ALP deviations of the L-PSIS and R-PSIS markers located at the posterior side of the pelvis also showed lower mean deviations $8.3 \pm 7.8 \mathrm{~mm}$ (Figure $5 \mathrm{c}$ ) to $12.4 \pm 8.9 \mathrm{~mm}$ (Figure $5 \mathrm{~b}$ ) compared to the markers located at the thoracic level STRN, L-AI, and R-AI, where mean deviations ranged from $16.1 \pm 9.7 \mathrm{~mm}$ (Figure $5 \mathrm{~b}$ ) to $38.6 \pm 23.1 \mathrm{~mm}$ (Figure 5c).

This indicates that during the rectification process, for all patients the location of the largest position changes was at the thoracic level. The ALP analysis showed smaller changes in pelvis landmark positions. This is likely because the orthotists use this location as a reference for the $3 \mathrm{D}$ alignment of the torso in the cranial/caudal direction during the rectification process.

\subsection{Surface Centroid Analysis}

For the different transverse sections along the patient body scans, the minimum average deviations from the reference system were located at the L-ASIS section (from $2.9 \pm 1.8 \mathrm{~mm}$ (medial/lateral) and $9.7 \pm 4.8 \mathrm{~mm}$ (anterior/posterior)) (Figure 6c), while maximum average deviations were found at the CLAV level (from $24.6 \pm 5.7 \mathrm{~mm}$ (me$\mathrm{dial} /$ lateral) and $36.2 \pm 30.4 \mathrm{~mm}$ (anterior/posterior)) (Figure 6f). The location of the minimum average deviations supports the findings from the ALP analysis and is likely due to the pelvis being used as a reference for the alignment during the manual rectification process (Figure $6 \mathrm{c}$ ). The average centroid deviations in the pre- and post-rectification mould were smaller than the deviations in the patient's body scan, indicating that, as expected, the deformity had been corrected to some extent during the casting and rectification processes (Figure 6g,h).

The results showed a rectification trend of the centroid trajectories towards the vertical Z-axis of the reference system located at the pelvis for all the patients throughout the casting and rectification steps. This trend suggests that centroid locations of transverse sections can be used to characterise the rectification process to some extent, with regard to the alignment of the torso with respect to the pelvis. Analysis of centroid locations using transverse sections could be used to define rotation parameters in the transverse plane, helping to improve the characterisation of the 3D spinal correction followed during the rectification process.

\subsection{Three-Dimensional Surface Deviation Analysis and Geometric Parameters}

The areas with the largest surface deviations were found on the convex side at the level of the CLAV, STRN, and superior of the iliac crest (ILIAC) in the medial/lateral direction (Figure 7f). The smallest surface deviations were located at the pelvis in the medial/lateral direction on the convex and concave sides (Figure 7f,g) and on the posterior side (Figure 7i). These results indicate that most of the rectification occurs on the convex side at the thoracic level, as it was observed in the ALP and centroid results, and at the iliac crest marker level corresponding to the location of the iliac crest pads. Deviations at the iliac crest and R-ASIS increased, corresponding to removal of material from the mould in the abdominal area, indicating a large anterior rectification in the sagittal plane (Figure $7 \mathrm{~h}$ ).

Rectification strategies were observed from lateral contours of the patient's body in the coronal plane, in terms of medial/lateral deviations and a torso tilt correction (Figure 8). The medial/lateral deviations at the waist level with respect to the pelvis width (L-ASIS to R-ASIS) showed a mean rectification ratio at the level of the iliac crest of $0.23 \pm 0.04$ on the 
convex side and $0.11 \pm 0.02$ on the concave side (Figure $8 \mathrm{i}$ ), therefore, demonstrating that this ratio can be used as a parameter for digital rectification. The lateral tilt angles from the external contours of the post-rectification mould were $81 \pm 3.8^{\circ}$ and $83.3 \pm 2.6^{\circ}$ on the convex and concave sides, respectively (Figure 8j). Moreover, the analysis of the contours in the sagittal plane showed mean tilt angles of $82.4 \pm 2.4^{\circ}$ (anterior) and $85.2 \pm 2.3^{\circ}$ (posterior) on the post-rectification moulds (Figure $8 \mathrm{k}$ ). The tilt angle results found in our study indicate rectification parameters that can be used to define the initial geometry of the rectified mould and the rectification distance at the iliac crest region, preceding the addition/removal of material corresponding to the pressure pad areas.

\subsection{Limitations}

Despite the small number of patients involved in the study $(n=5)$ and only TLSO designs being analysed, the sample size was large enough to show that characterisation of this process is possible. Demographics also present a major factor. This study analyses the data from Sri Lankan female patients from ages 11 to 16 years old, with right and left curves, and varying Risser grades. This range would have likely led to larger variability than had these factors been accounted for. Therefore, future work to elucidate trends in more controlled subsets of patients is warranted and may lead to improved quantification of the rectification process. A larger study on more brace designs and more curve types would be required before an algorithm can be developed for clinical use. Moreover, the characterisation results may vary depending on the orthotist involved in the manual rectification. To support the statistical significance of the found parameters, a larger number of patients and orthotists will need to be involved in a new study.

\section{Conclusions}

This study found two parameters that can be used to characterise important strategies of the mould rectification process for the design of scoliosis braces under the guidance of an orthotist using 3D scans of patients. First, the tilt angle on the lateral, anterior, and posterior sides was found as a parameter to define the external geometry inclination of the post-rectification mould in the coronal and sagittal plane. Second, the rectification ratio at the iliac crest level for the convex and concave side indicated the mean material removal with respect to the width of the patient's waist.

The characterisation parameters found in this study could be implemented into 3D sculpting software for the digital design of scoliosis braces, advancing towards the automation of the design process that will lead to more efficient additive manufacturing of scoliosis braces.

Author Contributions: Conceptualization, I.S.-P. and N.N.; methodology, I.S.-P. and N.N.; software, I.S.-P., S.A., and S.M.; validation, I.S.-P., N.N., D.H.-V., and J.K.-L.; formal analysis, I.S.-P. and N.N.; investigation, I.S.-P., S.A., and S.M.; resources, N.N., D.H.-V., J.K.-L., and P.S.; data curation, I.S.-P. and N.N.; writing—original draft preparation, I.S.-P. and N.N.; writing-review and editing, I.S.-P., S.A., D.H.-V., J.K.-L., N.N., and A.M.; visualization, I.S.-P. and N.N.; supervision, N.N., P.S., and A.M.; project administration, I.S.-P., S.A., and P.S.; funding acquisition, N.N. All authors have read and agreed to the published version of the manuscript.

Funding: This research was funded by Research England QR GCRF fund.

Institutional Review Board Statement: The study was conducted according to the guidelines of the Declaration of Helsinki and approved by the Institutional Review Board (or Ethics Committee) of SIDER from the Faculty of Medicine, University of Kelaniya, Ragama, Sri Lanka (FWA00013225 (REF. P/08/02/2020) approval date: 26/06/2020.

Informed Consent Statement: Informed written consent was obtained from the patients' parents/guardians involved in the study. Additional informed consent was obtained from the participant for whom identifying information is included in this article.

Data Availability Statement: The datasets generated during and/or analysed during the current study are available from the corresponding author on reasonable request. 
Conflicts of Interest: The authors declare no conflict of interest. The funders had no role in the design of the study; in the collection, analyses, or interpretation of data; in the writing of the manuscript, or in the decision to publish the results.

\section{References}

1. Kane, W.J. Scoliosis Prevalence: A Call for a Statement of Terms. Clin. Orthop. Relat. Res. 1977, 126, 43-46. [CrossRef]

2. Dickson, R.A. Scoliosis in the community. Br. Med. J. 1983, 286, 615-618. [CrossRef]

3. Weinstein, S. Adolescent idiopathic scoliosis: Prevalence and natural history. In The Pediatric Spine: Principles and Practice; Weinstein, S., Ed.; Raven Press: New York, NY, USA, 1994; pp. 463-478.

4. Soucacos, P.N.; Soucacos, P.K.; Zacharis, K.C.; Beris, A.E.; Xenakis, T.A. School-screening for scoliosis. A prospective epidemiological study in northwestern and central Greece. J. Bone Jt. Surg. Am. 1997, 79, 1498-1503. [CrossRef]

5. Pin, L.H.; Mo, L.Y.; Lin, L.; Hua, L.K.; Hui, H.P.; Hui, D.S.; Chang, B.D.; Chang, Y.Y.; Yuan, L. Early diagnosis of scoliosis based on school-screening. J. Bone Jt. Surg. Am. Vol. 1985, 67, 1202-1205. [CrossRef]

6. Schlenzka, D.; Yrjönen, T. Bracing in adolescent idiopathic scoliosis. J. Child. Orthop. 2013, 7, 51-55. [CrossRef]

7. Fayssoux, R.S.; Cho, R.H.; Herman, M.J. A History of Bracing for Idiopathic Scoliosis in North America. Clin. Orthop. Relat. Res. 2010, 468, 654-664. [CrossRef]

8. Emans, J.B.; Kaelin, A.; Bancel, P.; Hall, J.E.; Miller, M.E. The Boston Bracing System for Idiopathic Scoliosis: Follow-up Results in 295 Patients. Spine 1986, 11, 792-801. [CrossRef]

9. Allington, N.J.; Bowen, J.R. Adolescent idiopathic scoliosis: Treatment with the Wilmington brace. A comparison of full-time and part-time use. J. Bone Jt. Surg. Am. Vol. 1996, 78, 1056-1062. [CrossRef]

10. Piazza, M.R.; Bassett, G.S. Curve progression after treatment with the Wilmington brace for idiopathic scoliosis. J. Pediatr. Orthop. 1990, 10, 39-43. [CrossRef]

11. Watts, H.G.; Hall, J.E.; Stanish, W. The Boston brace system for the treatment of low thoracic and lumbar scoliosis by the use of a girdle without superstructure. Clin. Orthop. Relat. Res. 1977, 87-92. [CrossRef]

12. Weinstein, S.L.; Dolan, L.A.; Wright, J.G.; Dobbs, M.B. Effects of bracing in adolescents with idiopathic scoliosis. N. Engl. J. Med. 2013, 369, 1512-1521. [CrossRef]

13. Katz, D.E.; Herring, J.A.; Browne, R.H.; Kelly, D.M.; Birch, J.G. Brace wear control of curve progression in adolescent idiopathic scoliosis. J. Bone Jt. Surg. Am. Vol. 2010, 92, 1343-1352. [CrossRef] [PubMed]

14. Sanders, J.O.; Newton, P.O.; Browne, R.H.; Katz, D.E.; Birch, J.G.; Herring, J.A. Bracing for idiopathic scoliosis: How many patients require treatment to prevent one surgery? J. Bone Jt. Surg. Am. Vol. 2014, 96, 649-653. [CrossRef] [PubMed]

15. Sankar, W.N.; Albrektson, J.; Lerman, L.; Tolo, V.T.; Skaggs, D.L. Scoliosis in-brace curve correction and patient preference of CAD/CAM versus plaster molded TLSOs. J. Child. Orthop. 2007, 1, 345-349. [CrossRef] [PubMed]

16. Wong, M.S. Computer-aided design and computer-aided manufacture (CAD/CAM) system for construction of spinal orthosis for patients with adolescent idiopathic scoliosis. Physiother. Theory Pract. 2011, 27, 74-79. [CrossRef]

17. Lou, E.H.; Chan, A.C.Y.; Donauer, A.; Tilburn, M.; Hill, D.L. Ultrasound-assisted brace casting for adolescent idiopathic scoliosis, IRSSD Best research paper 2014. Scoliosis 2015, 10, 13. [CrossRef]

18. Cobetto, N.; Aubin, C.-E.; Clin, J.; Le May, S.; Desbiens-Blais, F.; Labelle, H.; Parent, S. Braces Optimized with Computer-Assisted Design and Simulations Are Lighter, More Comfortable, and More Efficient Than Plaster-Cast Braces for the Treatment of Adolescent Idiopathic Scoliosis. Spine Deform. 2014, 2, 276-284. [CrossRef]

19. Pazos, V.; Cheriet, F.; Danserau, J.; Ronsky, J.; Zernicke, R.; Labelle, H. Reliability of trunk shape measurements based on 3-D surface reconstructions. Eur. Spine J. 2007, 16, 1882-1891. [CrossRef]

20. Eldeeb, H.; Asfour, S.; Boubekri, N. CT/MR imaging: A design tool for custom orthosis. Disabil. Rehabil. 2000, 22, 583-590. [CrossRef]

21. De Santis, R.; Gloria, A.; Viglione, S.; Maietta, S.; Nappi, F.; Ambrosio, L.; Ronca, D. 3D laser scanning in conjunction with surface texturing to evaluate shift and reduction of the tibiofemoral contact area after meniscectomy. J. Mech. Behav. Biomed. Mater. 2018, 88, 41-47. [CrossRef]

22. Wong, M.S.; Cheng, J.C.Y.; Lo, K.H. A comparison of treatment effectiveness between the CAD/CAM method and the manual method for managing adolescent idiopathic scoliosis. Prosthet. Orthot. Int. 2005, 29, 105-111. [CrossRef]

23. Desbiens-Blais, F.; Clin, J.; Parent, S.; Labelle, H.; Aubin, C.-E. New brace design combining CAD/CAM and biomechanical simulation for the treatment of adolescent idiopathic scoliosis. Clin. Biomech. 2012, 27, 999-1005. [CrossRef]

24. Labelle, H.; Bellefleur, C.; Joncas, J.; Aubin, C.-E.; Cheriet, F. Preliminary evaluation of a computer-assisted tool for the design and adjustment of braces in idiopathic scoliosis: A prospective and randomized study. Spine 2007, 32, 835-843. [CrossRef]

25. Cottalorda, J.; Kohler, R.; Garin, C.; Genevois, P.; Lecante, C.; Berge, B. Orthoses for mild scoliosis: A prospective study comparing traditional plaster mold manufacturing with fast, noncontact, 3-dimensional acquisition. Spine 2005, 30, 399-405. [CrossRef]

26. Rigo, M.D.; Gallo, D.; Dallmayer, R. In-brace correction of the Cobb angle with RSC-CAD CAM compared with "hand made" from the original author. Scoliosis 2010, 5, O68. [CrossRef]

27. De Santis, R.; Gloria, A.; Russo, T.; D’Amora, U.; Zeppetelli, S.; Tampieri, A.; Herrmannsdörfer, T.; Ambrosio, L. A route toward the development of 3D magnetic scaffolds with tailored mechanical and morphological properties for hard tissue regeneration: Preliminary study. Virtual Phys. Prototyp. 2011, 6, 189-195. [CrossRef] 
28. Lanzotti, A.; Martorelli, M.; Maietta, S.; Gerbino, S.; Penta, F.; Gloria, A. A comparison between mechanical properties of specimens 3D printed with virgin and recycled PLA. Procedia Cirp 2019, 79, 143-146. [CrossRef]

29. Thometz, J.G.; Lamdan, R.; Liu, X.C.; Lyon, R. Relationship between Quantec measurement and Cobb angle in patients with idiopathic scoliosis. J. Pediatr. Orthop. 2000, 20, 512-516. [CrossRef]

30. Mínguez, M.F.; Buendía, M.; Cibrián, R.M.; Salvador, R.; Laguía, M.; Martín, A.; Gomar, F. Quantifier variables of the back surface deformity obtained with a noninvasive structured light method: Evaluation of their usefulness in idiopathic scoliosis diagnosis. Eur. Spine J. 2007, 16, 73-82. [CrossRef]

31. Adankon, M.M.; Chihab, N.; Dansereau, J.; Labelle, H.; Cheriet, F. Scoliosis follow-up using noninvasive trunk surface acquisition. IEEE Trans. Biomed. Eng. 2013, 60, 2262-2270. [CrossRef]

32. Klos, S.S.; Liu, X.-C.; Lyon, R.M.; Tassone, J.C.; Thometz, J.G. Reliability of a functional classification system in the monitoring of patients with idiopathic scoliosis. Spine 2007, 32, 1662-1666. [CrossRef]

33. Navarro, I.J.R.L.; da Rosa, B.N.; Candotti, C.T. Anatomical reference marks, evaluation parameters and reproducibility of surface topography for evaluating the adolescent idiopathic scoliosis: A systematic review with meta-analysis. Gait Posture 2019, 69, 112-120. [CrossRef] 